\title{
Interpretation of the secondary electromagnetic field by analytic continuation
}

\author{
M. Y. DizioğLU $\left(^{*}\right)$ \\ Ricevuto il 24 Giugno 1967
}

\begin{abstract}
SUMMARY. - Given the values of a component of secondary magnetic field on the plane surface of the earth, an attempt has been made to approach the sources by finding the values of the component on a plane at a depth $h$ below the surface, the plane being above the sources.

To this end a Green's Function for the wave equation, appropriate for a semi - infinite space was determined. The integral obtained was expanded into series suitable for numerical calculations.

The average values of the component on the earth plane were assumed to satisfy a double series and the coefficients were determined by the least square method.

Formulae were ultimately developed to calculate the values on a plane at a depth $h$, in terms of average values on six concentric circles around each point.
\end{abstract}

Riassunto. - Dati i valori di una componente di un campo magnetico secondario sulla superficie piana della Terra, si è tentato di individuare le sorgenti, trovando i valori della componente su un piano posto al di sopra delle sorgenti ad una profondità $h$ sotto la superficie.

A questo scopo è stata determinata una funzione di Green per l'equazione dell'onda, adattata ad uno spazio semi-infinito. L'integrale ottenuto \& stato esteso in serie, idonee ai calcoli numerici.

I valori medi della componente sul piano terrestre, sono stati assunti per soddisfare una serie doppia, ed i coefficienti sono stati determinati col metodo dei minimi quadrati.

Le formule sono state infine sviluppate per calcolare i valori sul piano alla profondità $h$, nei limiti dei valori medi, su sei cerchi concentrici attorno ad ogni punto.

(*) Chief Geophysicist - Mineral Research and Exploration Institute of Turkey. 
It is known that, with the inductive electromagnetic method of prospecting, the secondary magnetic field is investigated and thence some inferences as to the localities and position of the sources producing such fields are made $\left({ }^{1}\right)\left({ }^{2}\right)$. These interpretations are usually carried out by qualitative methods. Sometimes curve fitting methods are also used for this purpose, but such calculations are tedious and time consuming and different assumptions made for such calculations are usually far from the true state.

Here, in this paper an attempt is made to solve the revers problem. That is to say, given the components of the secondary magnetic field on the plane surface of the earth, we will try to approach the sources by finding the values of the components on a plane at a depth $h$ below the surface; the plane being above the sources.

It should be stated here that such a procedure would not at all improve the limitations of the method or lift the ambiguities of interpretation, which would remain the same $\left(^{3}\right)\left(^{4}\right)\left({ }^{5}\right)$. But such approaches towards the sources would enlarge the anomaly and subside the background, so that the resolving power of the interpretation would be increased.

Let us now assume that we know the components of the secondary field produced by a primary field uniform at every point. In practice such a secondary field can be obtained by keeping the distance between the antenna and the receiver and moving the arrangement at equal intervals along parallel lines.

These components would satisfy the equation (in Gaussian units):

$$
\nabla^{2} H_{i}=\frac{4 \pi \mu}{c^{2} \underline{\rho}} \cdot \frac{d H_{i}}{d t}+\frac{\mu k}{c^{2}} \cdot \frac{d^{n} H_{i}}{d t^{2}},
$$

where $H_{\imath}=$ any component of the secondary magnetic field

$$
\mu=\text { permeability of the medium }
$$$$
\varrho=\text { resistivity of the medium }
$$$$
k=\text { dielectric constant of the medium }
$$

$c=$ speed of light.

As, usually the resistivity of the country rock is quite high, the coefficient of the first term becomes negligibly small. Therefore it can be discarded.

If we assume that the primary field is sinusoidal, then:

$$
H_{\imath}=H(x, y, z) e^{i q c t},
$$


where,

$$
q=2 \pi f / c .
$$

The function $H$ must satisfy:

$$
\left(\nabla^{2}+k^{2}\right) H=0,
$$

where,

$$
k^{2}=\mu k q^{2}
$$

Approach towards the source:

Let any component of the secondary magnetic field be known on any plane $z=0$. This plane may be the surface of the earth or a plane above the surface of the earth as in the case of airborne electromagnetic prospecting. Let us suppose that $z$ is positive downwards in a direction towards the source of the field.

Let us define a function:

$$
\bar{H}(r, z)=\frac{1}{2 \pi} \int_{0}^{2 \pi} H(r, z, \theta) d \theta
$$

where $\bar{H}(r, z)$ is the average value of any particular component of the secondary magnetic field around a circle of radius $r$ centered at $(0,0, z)$.

If the $z$ axis does not pass through any source, it is possible to expand $\bar{H}(0, z)$ into series around $z=0$ in terms of the intensities on the surface of the earth in the form:

$$
\bar{H}(0, h)=\bar{H}(0,0)+h \frac{\partial \bar{H}(0,0)}{\partial z}+\frac{h^{2}}{2 !} \frac{\partial^{2} \bar{H}(0,0)}{\partial z^{2}}+\ldots \text { etc. }
$$

The derivatives in equation [2] can be calculated in different ways, but as the calculation of even derivatives are more direct than the calculation of odd ones, we can get rid of them by using the upward extrapolation:

$$
\bar{H}(0,-h)=\bar{H}(0,0)-h \frac{\partial \bar{H}(0,0)}{\partial z}+\frac{h^{2}}{2 !} \frac{\partial^{2} \bar{H}(0,0)}{\partial z^{2}}+\ldots \text { etc. }
$$

Upon adding equations [2] and [3], we obtain:

$$
\bar{H}(0, h)=2\left[\bar{H}(0,0)+\frac{h^{2}}{2 !} \frac{\partial^{2} \bar{H}(0,0)}{\partial z^{2}}+\ldots \text { etc. }\right]-\bar{H}(0,-h) .
$$


We have now to develop a method of calculation for the derivatives in brackets as well as for the last term $\bar{B}(0,-h)$ which is the continuation upward to a height $h$ above the surface. We will first deal with the calculation of the derivatives.

From the wave equation in polar coordinates:

$$
\begin{gathered}
\frac{\partial^{2} \bar{H}}{\partial z^{2}}=-\left(\frac{\partial^{2}}{\partial r^{2}}+\frac{1}{r} \frac{\partial}{\partial r}+k^{2}\right) \bar{H} \\
\frac{\partial^{4} \bar{H}}{\partial z^{4}}=\left(\frac{\partial^{2}}{\partial r^{2}}+\frac{1}{r} \frac{\partial}{\partial r}\right)^{2} \bar{H}+2 k^{2}\left(\frac{\partial^{2}}{\partial r^{2}}+\frac{1}{r} \frac{\partial}{\partial r}\right) \bar{H}+k^{4} \bar{H} \\
\frac{\partial^{6} \bar{B}}{\partial z^{6}}=-\left(\frac{\partial^{2}}{\partial r^{2}}+\frac{1}{r} \frac{\partial}{\partial r}\right)^{3} \bar{H}-3 k^{2}\left(\frac{\partial^{2}}{\partial r^{2}}+\frac{1}{r} \frac{\partial}{\partial r}\right)^{2} \bar{H}- \\
-3 k^{4}\left(\frac{\lambda^{2}}{\partial r^{2}}+\frac{1}{r} \cdot \frac{\partial}{\partial r}\right) \bar{H}-k^{6} \bar{H},
\end{gathered}
$$

where $\left(\frac{\partial-}{\partial r^{2}}+\frac{1}{r} \cdot \frac{\partial}{\partial r}\right)^{r}$, means that the operation is to be applied $i$ times in succession.

We can show by an application of Green's Theorem, that $\bar{H}(r, 0)$ is an even function, therefore it can be written in the form:

$$
\bar{H}(r, 0)=a_{0}+a_{2} r^{2}+a_{4} r^{4}+a_{6} r^{6}+\ldots \text { etc. }
$$

Now if we perform the operations for the derivatives, we find:

$$
\begin{gathered}
\frac{\partial^{2} \bar{H}(0,0)}{\partial z^{2}}=-\left(a_{0} k^{2}+4 a_{2}\right) \\
\frac{\partial^{4} \bar{H}(0,0)}{\partial \bar{z}^{4}}=a_{0} k^{4}+8 k^{2} a_{2}+64 a_{4} \\
\frac{\partial^{6} \bar{H}(0,0)}{\partial z^{6}}=-\left(a_{0} k^{6}+12 k^{4} a_{2}+192 k^{2} a_{4}+2304 a_{6}\right) .
\end{gathered}
$$

If we replace these in equation [4], we obtain:

$$
\begin{aligned}
& \bar{H}(0, h)=2\left[\bar{H}(0,0)-\frac{h^{2}}{2 !}\left(a_{0} k^{2}+4 a_{2}\right)+\frac{h^{4}}{4 !}\left(a_{0} k^{4}+8 k^{2} a_{2}+64 a_{4}\right)-\right. \\
& \left.-\frac{h}{6 !}\left(a_{0} k^{6}+12 k^{4} a_{2}+102 k^{2} a_{4}+2304 a_{6}\right)+\ldots \ldots\right]-\bar{H}(0,-h) .
\end{aligned}
$$


Since the convergence of the series in brackets is rapid enough, only four terms have been used.

Determination of the coefficients in the series [5].

We can take as many terms as we please, and obtain the values of their coefficients by means of the method of least squares. We have made two trials. In one of them we supposed that:

$$
\bar{H}(r, 0)=a_{0}+a_{2} r^{2}+a_{4} r^{4},
$$

and solved the normal equations with average values around circles of radius $0,1,2,3,4,5$ and 6 . The results are as follows:

$$
\begin{aligned}
a_{0}= & +0,386100 \bar{H}(0)+0,386100 \bar{H}(1)+0,250962 \bar{H}(2)+ \\
& +0,070772 \overline{\bar{H}}(3)-0,086910 \bar{H}(4)-0,127520 \bar{H}(5)+ \\
& +0,070610 \bar{H}(6)
\end{aligned}
$$

$$
\begin{aligned}
a_{2}= & -0,039186 \bar{H}(0)-0,039186 \bar{H}(1)-0,008508 \bar{H}(2)+ \\
& +0,029982 \bar{H}(3)+0,057324 \bar{H}(4)+0,046974 \bar{H}(5)- \\
& -0,035196 \bar{H}(6)
\end{aligned}
$$

$$
\begin{aligned}
a_{4}= & +0,000819 \bar{H}(0)+0,000819 \bar{H}(1)+0,000006 \bar{H}(2)- \\
& -0,000989 \bar{H}(3)-0,001626 \bar{H}(4)-0,001149 \bar{H}(5)+ \\
& +0,001414 \bar{H}(6) .
\end{aligned}
$$

If we assume that:

$$
\bar{H}(r, 0)=a_{0}+a_{2} r^{2}+a_{4} r^{4}+a_{6} r^{6},
$$

then the formulae for the coefficients found by the same method become:

$$
\begin{aligned}
a_{0}= & +0,02544 \bar{H}(0)+0,0220 \bar{H}(1)+0,1360 \bar{H}(2)- \\
& -0,00217 \bar{H}(3)+0,0970 \bar{B}(4)-0,0442 \bar{H}(5)- \\
& -0,030112 \bar{H}(6)+0,02616 \bar{H}(7) \\
a_{2}= & -0,000495 \bar{H}(0)-0,000361 \bar{H}(1)-0,000211 \bar{H}(2)+ \\
& +0,0000780 \bar{H}(3)-0,000256 \bar{H}(4)+0,000468 \bar{H}(5)+ \\
& +0,000343 \bar{H}(6)-0,000374 \bar{H}(7)
\end{aligned}
$$




$$
\begin{aligned}
a_{4}= & +0,0000143 \bar{H}(0)+0,0000186 \bar{H}(1)-0,0000195 \bar{H}(2)+ \\
& +0,0000721 \bar{H}(3)+0,0001825 \bar{H}(4)+0,000324 \bar{H}(5)+ \\
& +0,0001444 \bar{H}(6)-0,0002727 \bar{H}(7) \\
a_{5}= & +0,00000124 \bar{H}(0)+0,000000908 \bar{H}(1)-0,00000015 \bar{H}(2)- \\
& -0,00000191 \bar{H}(3)-0,00000378 \bar{H}(4)-0,00000679 \bar{H}(5)- \\
& -0,0000065 \bar{H}(6)+0,0000057 \bar{H}(7) .
\end{aligned}
$$

Evaluation of $\bar{H}(0,-h)$.

Let us suppose that the singularities of $\bar{H}$ all lie outside a closed surface $S$ bounding the volume $V$. Then putting:

$$
\bar{H}^{\prime}=\frac{e^{i k}\left|r-r^{\prime}\right|}{\left|r-r^{\prime}\right|},
$$

(where $r$ is the position vector of the point in consideration and $r^{\prime}$ is the position vector of any other point) and this value of $\bar{H}$ in the Green's Theorem and applying it to the region bounded externally by $S$ and internally by $C$, a small sphere with center $r$ and radius $\varepsilon$, we find that if the point $(P)$ lies inside $S$ :

$$
\begin{aligned}
& \int_{S}\left\{\bar{H}\left(r^{\prime}\right) \frac{\partial}{\partial n} \cdot \frac{e^{i k\left|r-r^{\prime}\right|}}{\left|r-r^{\prime}\right|}-\frac{e^{i k\left|r-r^{\prime}\right|}}{\left|r-r^{\prime}\right|} \cdot \frac{\partial \bar{H}\left(r^{\prime}\right)}{\partial n}\right\} d s^{\prime}= \\
= & \lim _{\varepsilon \rightarrow 0} \int_{\dot{C}}\left\{\left(i k-\frac{1}{\left|r-r^{\prime}\right|}\right) \bar{H}\left(r^{\prime}\right)-\frac{\partial \bar{H}\left(r^{\prime}\right)}{\partial r^{\prime}}\right\} \frac{e^{i k\left|r-r^{\prime}\right|}}{r-r^{\prime}} d s^{\prime} .
\end{aligned}
$$

We can show that the value of the limit on the right-hand side of this equation is $-4 \pi \bar{H}(r)$.

Determination of an appropriate Green's function for the half-space $z \leqslant 0$.

Let us suppose that $G\left(r, r^{\prime}\right)$ satisfies the same wave equation and that it is finite and continuous with respect to either the variables $x, y, z$ or to the variables $x^{\prime}, y^{\prime}, z^{\prime}$ for points $r, r^{\prime}$ belonging to a region $V$ which is bounded by a closed surface $S$ except in the neighbourhood of the point $r$, where it has a singularity of the same type as:

$$
\frac{e^{i k\left|r-r^{\prime}\right|}}{\left|r-r^{\prime}\right|}
$$


as $r^{\prime} \rightarrow r$. Then proceeding as in the derivation of equation [16], we can prove that, if $r$ is the position vector of a point within $V$, then:

$$
\bar{H}(r)=\frac{1}{4 \pi} \int_{S}\left\{G\left(r, r^{\prime}\right) \frac{\partial \bar{H}\left(r^{\prime}\right)}{\partial n}-\bar{H}\left(r^{\prime}\right) \frac{\partial G\left(r, r^{\prime}\right)}{\partial n}\right\} d s^{\prime} .
$$

where $n$ is the outward-drawn normal to the surface $S$.

It follows immediately from equation [17] that if $G_{1}\left(r, r^{\prime}\right)$ is such that it satisfies the boundary condition:

$$
G_{1}\left(r, r^{\prime}\right)=0
$$

and if the point with position vector $r^{\prime}$ lies on $S$, then:

$$
\bar{H}(r)=-\frac{1}{4 \pi} \int_{S} \bar{H}\left(r^{\prime}\right) \frac{\partial G_{1}\left(r, r^{\prime}\right)}{\partial n} d s^{\prime} .
$$

Now for the half-space:

$$
G_{1}\left(r, r^{\prime}\right)=\frac{e^{|k| r-r^{\prime} \mid}}{\left|r-r^{\prime}\right|}-\frac{e^{i k\left|2-r^{\prime}\right|}}{\left|l-r^{\prime}\right|}
$$

where $l=(x, y, z)$ is the position vector of the image in the plane $z-0$ of the point with position vector $r=(x, y,-z)$. For this function it is easily shown that if the point with position vector $r^{\prime}$ lies on $N$, the $x, y$ plane, then:

$$
\frac{\partial G_{1}}{\partial n}=\frac{\partial G_{1}}{\partial z^{\prime}}=-2-\frac{\partial}{\partial z}\left(\frac{e^{i k R}}{R}\right)
$$

where:

$$
R^{2}=\left(x-x^{\prime}\right)^{2}+\left(y-y^{\prime}\right)^{2}+z^{2} .
$$

It follows from equation [18] that if $\bar{H}=f(x, y)$ on $z=0$, then when $z<0$ :

$$
\bar{H}(x, y,-z)=\frac{1}{2 \pi} \frac{\partial}{\partial z} \int_{\dot{N}} f\left(x^{\prime}, y^{\prime}\right) \frac{e^{i k R}}{R} d x^{\prime} \cdot d y^{\prime}
$$

where $\bar{H}(x, y,-z)$ is the value of a component of the secondary field at the point $(x, y,-z)$ above the surface.

Since the measured field is not known as a mathematical function, we wish to devise a practical scheme for the numerical evaluation of the integral. 
Evaluation of the integral.

Putting the real component of $\frac{\partial}{\partial z} \frac{e^{(k R}}{R}$ in the integral;

$$
\begin{aligned}
& \bar{H}(x, y,-z)=-\frac{z}{2 \pi} \int_{-\infty}^{+\infty} \int_{-\infty}^{-\infty} f\left(x^{\prime}, y^{\prime}\right) \frac{\cos k R+k R \sin k R}{R^{3}} d x^{\prime} \cdot d y^{\prime}, \\
& \quad \text { let } \quad\left(x^{\prime}-x\right)=r \cos \theta ; \quad\left(y^{\prime}-y\right)=r \sin \theta \\
& \text { since } \\
& \quad d x^{\prime} \cdot d y^{\prime}=\left|7\left(\frac{x y}{r \theta}\right)\right| d r \cdot d \theta=r d r \cdot d \theta . \\
& \bar{H}=-\frac{z}{2} \pi \int_{0}^{r=\infty} \int_{0}^{0=2 \pi} F(r, \theta) \frac{\cos k R+k R \sin k R}{R^{3}} r d r d \theta .
\end{aligned}
$$

If we define a function:

$$
\bar{H}_{z}(r)=\frac{1}{2 \pi} \int_{0}^{2 \pi} H_{z}(r, \theta) d \theta \text {, }
$$

the equation [20] becomes:

$$
\bar{H}=-\int_{i j}^{r=\infty} \bar{H}(r) \frac{\cos k R+k R \sin k R}{R^{3}} r \cdot z \cdot d r,
$$

this can be written in the form:

$$
\begin{gathered}
\bar{H}=\frac{\bar{H}(0)+\bar{H}\left(b_{1}\right)}{2} z \int_{0}^{b_{1}} \frac{\cos k R+k R \sin k R}{k^{3}} r \cdot d r+ \\
+\frac{\bar{H}\left(b_{1}\right)+\bar{H}\left(b_{2}\right)}{2} z \int_{b_{1}}^{b_{2}} \frac{\cos k R+k R \sin k R}{R^{3}} r \cdot d r+\text { etc. }
\end{gathered}
$$

where $b_{1} \cdot b_{2} \ldots$ are the radii of circles around the origin. 
If we expand $\cos k R$ and $\sin k R$ and make the necessary integrations, we obtain the following series:

$$
\begin{aligned}
& \bar{H}(0,-h)=h \frac{\bar{H}(0)}{2}\left\{\left[\frac{1}{h}-\frac{1}{\left(b_{1}^{2}+h^{2}\right)^{1 / 2}}\right\rceil-\mid h-\left(b_{1}^{2}+h^{2}\right)^{1 / 2}\right\rceil \\
& k^{2}\left(1-\frac{1}{2 !}\right)-\left|h^{3}-\left(b_{1}^{2}+h^{2}\right)^{3 / 2}\right| \frac{k^{4}}{3}\left(\frac{1}{3 !}-\frac{1}{4 !}\right)+ \\
& \left.+\left\lfloor h^{3}-\left(b_{1}^{2}+h^{2}\right)^{5 / 2}\right] \mid \frac{k^{6}}{5}\left(\frac{1}{5 !}-\frac{1}{6 !}\right)-\ldots\right\}+ \\
& +h \frac{\bar{H}\left(b_{1}\right)}{2} \text { [replace } b_{1} \text { by } b_{2} \text { in the same series] + } \\
& +h \frac{\bar{H}\left(b_{2}\right)}{2}\left\{\left[\frac{1}{\left(b_{1}^{2}+h^{2}\right)^{/ 1_{2}}}-\frac{1}{\left(b_{2}^{2}+h^{2}\right)^{1 / 2}}\right]-\right. \\
& -\left[\left(b_{1}^{2}+h^{2}\right)^{1 / 2}-\left(b_{0}^{2}+h^{2}\right)^{1 / 2} \mid k^{2}\left(1-\frac{1}{2 !}\right) \cdots\right\}+h \frac{\bar{H}\left(b_{3}\right)}{2}
\end{aligned}
$$

[replace $\bar{b}_{1}$ and $b_{2}$ by $b_{2}$ and $b_{3}$ respectively in the last series] $+\ldots$ etc. [22]

By replacing this formula in equation [6], the complete formula for the evaluation of the intensity at a depth $h$ can be obtained. By this formula, it would be possible to find the required intensities on a plane surface nearer the sources thus the localities of the sources producing the secondary field could be inferred.

\section{REFERENCES}

(1) WesceY J. P., Response of a dyke to an oscillating dipole. "Geophysics", 23, pp. 128-133 (1958).

${ }^{2}$ ) WAIT J. R., A conducting sphere in a time varying magnetic field. "Geophysics ", 16, pp. 666-672 (1951).

( ${ }^{3}$ SLIChter L. B., An inverse boundary value problem in electrodynamics. "Physics ", 4, p. 418 (1933).

(1) SkeELs D. C.. Ambiguity in gravity interpretation. "Goophysics ", 12, pp. 43.56 (1947).

${ }^{5}$ ) Tsubor C. and Fochida T., Relations between gravity values and corresponding subterranean mass distributions. "Bulletin of the Earthquake Research Institute ", Tokyo, Imperial University, 15, pp. 636-649 (1937). 